\title{
El desarrollo de las competencias orales en el Grado en Derecho a través de la participación en competiciones de arbitraje (moot courts) ${ }^{1}$
}

The development of oral skills in the Law Degree through the participation in arbitration competitions (moot courts).

\author{
Silvia Madalina Boboc ${ }^{* *}$ \\ Instituto Universitario de Derecho del Transporte \\ Universitat Jaume I, España \\ E-mail: $\underline{\text { boboc@uji.es }}$ \\ Ciara Vicente Mampel \\ Instituto Universitario de Derecho del Transporte \\ Universitat Jaume I, España \\ E-mail: vicentec@uji.es
}

\begin{abstract}
Resumen: En el marco del proceso de aprendizaje basado en competencias y su implantación en el Grado en Derecho de la Universitat Jaume I, la Competición Internacional de Arbitraje y Derecho Mercantil (en adelante, MOOTmadrid), organizada por la Universidad Carlos III de Madrid y la Comisión de las Naciones Unidas para el Derecho Mercantil Internacional (CNUDMI/UNCITRAL) constituye una herramienta didáctica adecuada para la adquisición o el desarrollo de algunas de las competencias propias de la titulación, entre las que ocupan un lugar destacado las competencias orales. Así, el presente artículo analiza el desarrollo de la capacidad oral a través de la participación de los estudiantes en la simulación de procedimientos arbitrales, utilizando para ello un cuestionario de opinión de elaboración propia, para
\end{abstract}

\footnotetext{
${ }^{1}$ El presente trabajo se ha realizado en el marco del Proyecto de Innovación Educativa «MOOTmadrid: Competición Internacional de Arbitraje y Derecho Mercantil», financiado por la Unidad de Soporte Educativo de la Universitat Jaume I de Castellón (ref. 3659/18). Investigadores principales: M. ${ }^{a}$ Victoria Petit Lavall y A. Puetz.

** Las dos autoras han contribuido en igual medida en la elaboración del trabajo.
} 
realizar a continuación propuestas de mejora en la metodología docente con el fin de potenciar el apropiado desarrollo de dichas competencias de cara a ediciones futuras de la competición.

Palabras clave: aprendizaje basado en competencias, competencias orales, moot courts, cuestionario de opinión, metodología docente.

Abstract: The International Competition of Arbitration and Commercial Law (hereinafter, MOOTmadrid), organized by the Carlos III University of Madrid and the United Nations Commission on International Trade Law (CNUDMI/UNCITRAL) represents an appropriate educational tool in order to acquire or develop certain basic competences required by the implementation of the competence-based learning process in the Degree in Law of the Jaume I University. Oral skills are a prime example amongst these competences. Thereby, this paper assesses the development of oral skills acquired by the students through their participation in arbitral proceedings simulations. An opinion questionnaire has been employed for that purpose. Subsequently, proposals for improving the teaching methodology are made, with a view to enhance the proper development of these skills for future editions of the competition.

Keywords: competence-based learning, oral skills, moot courts, opinion questionnaire, teaching methodology.

\section{Introducción}

La simulación de juicios y procedimientos arbitrales se introdujo en la enseñanza del Derecho a finales del siglo XVI, en la práctica de las asociaciones profesionales de barristers y solicitors en Gran Bretaña llamadas Inns of Court e Inns of Chancery, siendo posteriormente desarrollada a finales del siglo XVIII en el marco de la educación jurídica formal en los Estados Unidos, donde dio lugar a una asentada tradición de moot practice (AÑÓN, 2014, 164; MARTINEAU, 1989, 71-86; RACHID y KNERR, 2000)². En estas actividades, el arte de convencer, esto es, de articular con claridad una postura jurídica, es de lo más relevante (GRAVES y VAUGHAN, 2006, 191). Dicho en otras

\footnotetext{
${ }^{2}$ En el sistema judicial británico y también en el australiano, los barristers son abogados que pueden actuar en todas las instancias judiciales, mientras que los solicitors actúan tan solo en las instancias inferiores (vid. Cambridge Dictionary, https://dictionary.cambridge.org/es/).
} 
palabras, la participación de los estudiantes universitarios en competiciones de arbitraje resulta ser una herramienta didáctica idónea para desarrollar, o al menos mejorar, su capacidad de comunicación oral.

Si bien en la actualidad existen distintas iniciativas en la disciplina del Derecho mercantil que se basan en las experiencias de los moot courts (ARROYO VENDRELL, 2016, 37-40; PERALES VISCASILLAS, 2016, 251-254), la Competición Internacional de Arbitraje y Derecho Mercantil (en adelante, MOOTmadrid), organizada por la Universidad Carlos III de Madrid y la Comisión de las Naciones Unidas para el Derecho Mercantil Internacional (CNUDMI/UNCITRAL), en la que la Universitat Jaume I lleva participando desde su sexta edición, celebrada en el año 2014, es una de las de mayor calado a nivel nacional (sobre las experiencias de la participación de la Universitat Jaume I en distintas ediciones del MOOTtmadrid, véase PUETZ, 2015, 396406; BOBOC, 2018). Se trata de una iniciativa basada en el aprendizaje por proyectos que permite abordar cuestiones relacionadas con el Derecho mercantil internacional desde una visión pragmática e instrumentalizada, a través de la simulación de procedimientos arbitrales, que sitúa al alumnado en dos posiciones contrapuestas (demandante y demandado) para la resolución de una controversia concreta jurídicamente fundada. Esta iniciativa persigue la formación de futuros abogados con perfil internacional, al potenciar el conocimiento y uso de textos legales de Derecho uniforme del comercio internacional y el recurso al arbitraje comercial internacional como medio usual en la resolución de disputas en la contratación mercantil (Bases y reglas de la Competición Internacional de Arbitraje y Derecho mercantil, s.f., 3). Para ello, se tratan dos aspectos de la disciplina jurídica claramente diferenciados: por un lado, el aspecto procesal que versa sobre el arbitraje como forma alternativa de resolución de controversias y, por otro lado, el aspecto sustantivo que se limita a supuestos de hecho referidos al Derecho mercantil (PUETZ, 2015, 400).

Pues bien, el objetivo del presente artículo consiste precisamente en valorar la adquisición de competencias orales por los estudiantes del Grado en Derecho de la Universitat Jaume I, a través de su participación en esta competición de arbitraje, y realizar a continuación propuestas de mejora en la metodología docente con el fin de potenciar la adquisición de dichas competencias de cara a ediciones futuras de la competición. El punto de partida para tal cometido lo constituye necesariamente el proceso de enseñanza-aprendizaje basado en competencias y su implantación en el 
Grado en Derecho de la Universitat Jaume I. En este contexto, la participación de los alumnos en el MOOTmadrid se presenta como una herramienta didáctica adecuada para la adquisición de algunas de las competencias propias de la titulación, especialmente aquellas relacionadas con la oratoria jurídica (punto 2). Seguidamente, se detalla el funcionamiento de la fase oral de la competición y los métodos de preparación de los estudiantes para su intervención en las rondas orales (punto 3). A continuación, se evalúan las competencias orales adquiridas por el alumnado, utilizando para ello un cuestionario de opinión de elaboración propia (punto 4), que se adjunta al final del presente trabajo como Anexo único. Finalmente, se efectúa una serie de propuestas de mejora en la metodología docente, cuya implantación se prevé que se llevará a cabo en futuras ediciones de la competición (punto 5).

\section{La implantación del aprendizaje basado en competencias en el Grado en} Derecho de la Universitat Jaume I. El MOOTmadrid como herramienta didáctica para la adquisición de competencias orales

El aprendizaje basado en competencias, entendido como un proceso de enseñanzaaprendizaje centrado en la capacidad y responsabilidad del estudiante y en el desarrollo de su autonomía, es uno de los ejes principales de la ya notoria actuación de la Unión Europea en el ámbito educativo, consistente en el establecimiento del llamado Espacio Europeo de Educación Superior. La integración de este proceso en la educación universitaria ha exigido una adecuación de los planes de estudios, de forma que éstos partan de un perfil académico-profesional que recoja los conocimientos y las competencias que se desea que los estudiantes desarrollen y alcancen en el título de Grado en Derecho, ya sean éstas genéricas o específicas (VILLA y POBLETE, 2007, $27-33)^{3}$.

El Plan de estudios del Grado en Derecho de la Universitat Jaume I prevé, en este sentido, unos contenidos que persiguen dotar al estudiante de una formación jurídica sólida para el desarrollo de las competencias necesarias para su inmersión profesional

\footnotetext{
${ }^{3}$ Se entiende que el desarrollo de estas competencias permite a los estudiantes adquirir los conocimientos científicos y técnicos necesarios y que éstos sean capaces, además, de aplicar los conocimientos adquiridos en contextos diversos y complejos, integrarlos con sus propias actitudes y valores en un modo propio de actuar tanto personal como profesionalmente. Por ello, este modelo de aprendizaje encuentra su fundamento principal en el análisis de las exigencias profesionales, que ayudan a definir y priorizar las competencias fundamentales requeridas en una determinada área profesional y/o de especialidad.
} 
en cualquiera de los ámbitos a los que habilita la titulación ${ }^{4}$. Destaca, entre ellas, el desarrollo de la oratoria jurídica, esto es, de la capacidad para expresarse apropiadamente ante un auditorio (E26) (RAMÍREZ RAMÍREZ, 2002, 60). Ciertamente, el profesional del Derecho debe mostrar un perfil competencial marcado por la adquisición tanto de unas aptitudes de superación permanente, aprendizaje continuo y flexibilidad, como también de unas habilidades o destrezas como la oratoria, la autoridad, la capacidad directiva y negociadora, la planificación y convicción jurídica (Memoria del Título de Graduado o Graduada en Derecho por la Universitat Jaume I, 2013, 14). De hecho, la adquisición de destrezas lingüísticas, como es el caso de la capacidad de comunicación oral (oratoria y retórica jurídica), constituye una competencia altamente considerada por los profesionales del Derecho para el acceso al mercado laboral (ANECA, 2005, 108 y ss.; GOÑI y MESEGUER, 2010, 42). No obstante, debe destacarse que su consecución plantea numerosos retos docentes, pues el desarrollo de habilidades de expresión oral presenta deficiencias no sólo en el ámbito de la educación superior, sino también en todos los niveles del sistema educativo (RAMÍREZ RAMÍREZ, 2002, 64 y ss.).

En efecto, la metodología que se ha venido empleando en las ciencias jurídicas no ha sido distinta en lo esencial a la que tradicionalmente se ha desarrollado en toda la enseñanza universitaria: la denominada «lección magistral» (hoy día, «lección expositiva»). Sin embargo, si lo que se busca es orientar la docencia universitaria hacia la práctica, esta metodología no suele considerarse el procedimiento más eficaz, dado que sólo con ella no se logra una participación activa del alumnado en su proceso de aprendizaje (DE MIGUEL, 2006, 22). Por el contrario, el proceso de aprendizaje podría quedar reducido a un plano estrictamente teórico o exegético ${ }^{5}$. De ahí que, siguiendo los planteamientos que inspiran el proceso europeo de convergencia de la educación

\footnotetext{
${ }^{4}$ Memoria del Título de Graduado o Graduada en Derecho por la Universitat Jaume I (2013), 12 y ss., https://documents.uji.es/alfresco/d/d/workspace/SpacesStore/bbc80171-53e8-46a8-be50-

782ca1c83c1e/Memoria+modificada+por+ANECA+261213+Derecho.pdf?guest=true. La Memoria no sólo incluye las competencias básicas recogidas en el Real Decreto 1393/2007, de 29 de octubre, por el que se establece la ordenación de las enseñanzas universitarias oficiales (B.O.E n. ${ }^{\circ} 260$, de 30 de octubre de 2007), sino que, además, éstas se concretan en una serie de competencias genéricas y/o específicas correspondientes a los objetivos que se deben alcanzar para la obtención del título de Graduado o Graduada en Derecho. En relación con las competencias genéricas destaca, por ejemplo, el aprendizaje autónomo, la capacidad de análisis y síntesis o la capacidad de organización y planificación. Las competencias específicas, por su parte, se refieren a la adquisición de una conciencia crítica en el análisis del ordenamiento jurídico y el desarrollo de la dialéctica jurídica, entre otras.

${ }^{5}$ A menos que la exposición no fuera únicamente unidireccional, sino que se viera completada, además, con la apertura de una fase de debate entre el docente y los estudiantes de modo bidireccional. Sobre las clases teóricas o explicaciones (lección magistral) como estrategia metodológica en Derecho mercantil, véase VEGA VEGA, 2009, 43-47.
} 
superior, haya sido necesaria la renovación de las metodologías de enseñanza tradicionales para adaptarlas a los nuevos planes de estudios basados en la adquisición de competencias, mediante la incorporación de métodos de trabajo cooperativo y basados en proyectos que integran actividades vinculadas al mundo de la práctica.

En relación a la capacidad de comunicación oral, destacan las simulaciones de procedimientos arbitrales entre las distintas herramientas disponibles ${ }^{6}$. En efecto, a través de su participación en competiciones que emulan el funcionamiento de los tribunales arbitrales - típico ejemplo de aprendizaje basado en proyectos- los estudiantes del Grado en Derecho de la Universitat Jaume I desarrollan, o al menos mejoran cualitativamente, alguna de las aptitudes necesarias para su adecuado desarrollo profesional en cualquiera de los ámbitos a los que habilita la titulación, entre ellas, la capacidad de comunicación oral ${ }^{7}$. Ello se debe a que este método de enseñanza exige que el alumno estudie en profundidad un caso complejo y busque soluciones jurídicamente bien fundadas $y$, además, desarrolle una estrategia procesal que, aparte de reflejarse en los escritos de demanda y contestación, también debe defenderse en la fase oral de la competición ${ }^{8}$.

\section{EI desarrollo y la preparación de la fase oral del MOOTmadrid}

\subsection{El desarrollo de la fase oral}

La fase oral de la XI edición de la competición MOOTmadrid tuvo lugar, como su propio nombre lo sugiere, en la ciudad de Madrid, entre el 1 y el 5 de abril 2019. Esta fase se compone de rondas generales en las que participan todos los equipos y rondas eliminatorias, esto es, cuartos de final y semifinales, culminando con la ronda final. En

\footnotetext{
${ }^{6}$ Las simulaciones de juicios constituyen también una herramienta didáctica válida para la adquisición o mejora de competencias orales. Algunas experiencias prácticas en relación con la simulación de juicios como metodología docente del Grado en Derecho pueden ser consultadas en ARMENGOT VILAPLANA, 2016, 33-36; DíAZ PITA, 2016, 97-100 y SÁNCHEZ SÁNCHEZ, 2016, 293-296. Otras herramientas destinadas al desarrollo de las competencias orales son las exposiciones orales y los debates. En cuanto a estos últimos, resulta significativa la experiencia relativa a la celebración de un debate universitario y multidisciplinar que relata CAÑABETE PÉREZ, 2016, 53-56.

${ }^{7}$ Coinciden en considerar la práctica de los moot courts como una herramienta fundamental para la preparación de los futuros profesionales del Derecho, GRAVES y VAUGHAN, 2006, 175; GABRIEL, 1999, 580. Es reveladora la afirmación de este último, quien considera que hasta los abogados más experimentados que trabajan para el Departamento de Justicia de los Estados Unidos necesitan practicar las sesiones de moot court antes de actuar en sala.

${ }^{8}$ Éste ha sido el planteamiento de los entrenadores desde que el primer equipo de la Universitat Jaume I participara en la competición de arbitraje MOOTmadrid, mucho antes de que las autoras del presente artículo se les unieran, PUETZ, 2016.
} 
las rondas generales cada equipo compite cuatro veces: dos como demandante y dos como demandado.

Cada ronda tiene una duración máxima de dos horas, durante las cuales se le asigna a cada orador una intervención de entre 15 y 25 minutos. Durante esta fase, los oradores, que no pueden ser más de dos por audiencia, representan a sus respectivos equipos frente al resto de participantes, viéndose alternadas las posiciones de demandante y demandado. De este modo, el número máximo de oradores por equipo es de 8 . No obstante, debe destacarse que sólo podrán optar al premio al mejor orador aquellos que hayan intervenido ante el tribunal arbitral tanto en calidad de demandante como de demandado (Bases y reglas de la Competición Internacional de Arbitraje y Derecho mercantil, s.f., 8).

Por su parte, la dirección del procedimiento corre a cargo de los árbitros, que gozan de discrecionalidad para establecer todo tipo de cuestiones relativas al procedimiento arbitral, decidiendo sobre el orden y los tiempos de cada intervención, los turnos de réplica y dúplica, pudiendo incluso interrumpir la disertación de los oradores si así lo consideran pertinente. Dicha discrecionalidad no implica, sin embargo, arbitrariedad, pues la actuación de los árbitros debe basarse en estrictos criterios de equidad. Asimismo, son los encargados de valorar la actuación de cada equipo participante y asignarle una puntuación en cada una de las cuatro rondas orales. La suma de dichas puntuaciones determinará la posición final del equipo y, en su caso, el pase a las rondas eliminatorias de la competición (Bases y reglas de la Competición Internacional de Arbitraje y Derecho mercantil, s.f., 8).

\subsection{La metodología empleada para la preparación de las rondas orales}

La metodología empleada para la preparación de las rondas orales de la competición MOOTmadrid del presente año no ha sido distinta a la que se ha venido empleando en el resto de ediciones. En primer lugar, cada uno de los miembros del equipo debe redactar, de manera individual, un resumen del caso, destacando en él la parte que más había sido trabajada en la fase escrita. Una vez preparados los resúmenes, los integrantes del equipo los exponen individualmente ante los compañeros y entrenadores, que a continuación eligen por unanimidad a los representantes del equipo en la competición. Así, se eligen cuatro estudiantes: dos para actuar en la posición del demandante, defendiendo uno de ellos la parte de Derecho sustantivo y el otro la de 
Derecho procesal; y dos en la posición del demandado, con la misma distribución. Bajo el mismo criterio de selección, se asigna a cada orador principal un suplente, con la finalidad de cubrir cualquier posibilidad de vacante. Esta estrategia ha resultado ser acertada, pues en algunas ediciones los suplentes han acabado siendo los oradores principales en las rondas orales, por distintas circunstancias.

A continuación, se asignan distintas tareas o roles a los miembros del equipo, ya sean oradores o no. En concreto, mientras que los primeros se dedican a preparar la intervención en las rondas orales, los no oradores estudian con detalle los escritos de demanda y contestación a la demanda del resto de participantes en la competición, con el fin de identificar eventuales errores o elaborar contraargumentos que desvirtúen las posibles posiciones de la contraparte en cada una de las audiencias. Una vez asignados los roles a cada uno de los integrantes del equipo, las reuniones suelen adquirir la dinámica: los cuatro oradores y sus respectivos suplentes realizan reuniones semanales en las que cada uno prepara su actuación y la expone frente al resto; también con carácter semanal, se realizan reuniones del equipo completo y los entrenadores; en un aula que simula una sala de vistas, los oradores exponen los resultados de su trabajo y emulan la intervención oral, que es, a continuación, revisada mediante la autoevaluación y el debate colectivo.

Por un lado, la autoevaluación permite a los alumnos identificar los aciertos y los errores que han podido detectar durante su intervención. A través del debate colectivo, por otro lado, se efectúa una «evaluación por pares» («peer-review»), que contribuye a que todos los estudiantes adopten una actitud activa en el proceso de enseñanzaaprendizaje. Junto con estas dos técnicas de evaluación, los entrenadores proporcionan feedback a los estudiantes y plantean sugerencias y mejoras que éstos deben adoptar en sus intervenciones. Se busca, en definitiva, ajustar paulatinamente los tiempos de exposición de cada una de las intervenciones, al tiempo que desarrollan una competencia comunicativa aceptable que incluya aptitudes tanto expresivas como comprensivas. Ello se debe a que, como es sabido, un buen comunicador debe adoptar una actitud activa, mirar e interactuar con el orador, ser objetivo, respetuoso, analítico y crítico, al tiempo que planifica el discurso, adecua la tonalidad, respeta los principios de textualidad y cooperación comunicativa, cuida la imagen y los gestos que forman el discurso, y observa las reacciones de la audiencia (RAMÍREZ RAMÍREZ, 2002, 60). En otras palabras, no sólo se trata de mejorar la actuación en sala de los estudiantes 
(actitud activa, interactiva, analítica, crítica y respetuosa), sino también y sobre todo la planificación del discurso, las técnicas argumentativas, la entonación y el lenguaje no verbal empleados en las intervenciones orales.

\section{La evaluación del desarrollo de las competencias orales sobre la base de un cuestionario de opinión}

\subsection{La muestra seleccionada}

Para evaluar la adquisición o la mejora de las competencias orales de los estudiantes del Grado en Derecho debidas a su participación en el MOOTmadrid se ha elaborado un cuestionario compuesto por 11 preguntas a contestar en una escala de 1 a 5 , donde «1» significa «nada» y 5 significa «totalmente». Asimismo, el cuestionario cuenta con una rúbrica final donde los estudiantes pueden incluir sus opiniones, reflexiones o ideas de forma libre (vid., con detalle, Anexo I).

Por lo que respecta a la muestra seleccionada, el cuestionario se envió a un total de 29 estudiantes, principalmente participantes en las últimas dos ediciones de la competición, pero también a 4 participantes en la edición correspondiente al año 2015 y a un participante de la edición del año 2014. La selección de la muestra se justifica porque se presumió que los participantes de las últimas dos ediciones tendrían aún recientes las vivencias relacionadas con su participación en el MOOTmadrid y que, por tanto, sus respuestas serían fiables y no estarían viciadas por el paso del tiempo. Por lo que respecta a los participantes del año 2015, se les envió el cuestionario porque una de las autoras del presente artículo seguía manteniendo contacto con ellos, después de haber formado parte ella misma del equipo de la Universitat Jaume I en dicha edición. Se trataba, por tanto, de una buena oportunidad para ampliar la muestra y así obtener resultados más relevantes. Finalmente, el cuestionario también fue enviado a un compañero del área de Derecho constitucional, actualmente investigador en formación, y que había participado en la edición del MOOTmadrid del año 2014. Sus aportaciones las consideramos especialmente relevantes, ya que se dedica a una labor en la que el empleo de las capacidades orales es significativo. Pues bien, de entre los alumnos a los que se les envió la encuesta, fueron doce los que la contestaron: 24 de las últimas dos ediciones, 4 de la edición de 2015 y 1 de la edición de 2014.

\subsection{Los resultados obtenidos}


A fin de conseguir una mejor claridad expositiva, los resultados obtenidos se explicarán en dos partes: en primer lugar, se referirán de forma conjunta los resultados obtenidos en 11 de las encuestas recibidas y, después, se detallarán las respuestas proporcionadas por uno de los participantes, ya que su percepción se aparta del sentir general expresado por los estudiantes.

En el primer caso, la mejora de las capacidades evaluadas a través del cuestionario se ha valorado por los encuestados según se detalla a continuación; la capacidad para identificar, analizar y resolver situaciones prácticas desde un punto de vista jurídico (pregunta n. ${ }^{\circ}$ ), ha obtenido 9 valoraciones de 5 estrellas y 2 de 4 estrellas; la capacidad para organizar el discurso oral (pregunta $n .^{\circ} 2$ ) ha sido valorada con 5 estrellas en 8 de los cuestionarios contestados y con 4 estrellas en 3 de ellos; la mejora de la capacidad de interacción oral (pregunta $n^{\circ}$ 3) ha sido puntuada con 5 estrella por 7 de los encuestados y con 4 estrellas por 4 de ellos; mientras que la mejora de la capacidad de argumentación (pregunta n. ${ }^{\circ}$ ) ha obtenido una puntuación de 5 estrellas en 7 de los casos, 3 de 4 estrellas y una de 3 estrellas; la capacidad para gestionar adecuadamente el tiempo en las intervenciones orales (pregunta $n^{\circ}{ }^{\circ}$ ) ha sido puntuada con 5 estrellas por 7 participantes y con 4 estrellas por 4 de ellos; la capacidad para trabajar bajo presión (pregunta n. ${ }^{\circ}$ ) ha obtenido 7 puntuaciones de 5 estrellas, 2 de 4 estrellas y 2 de 3 estrellas; la mejora de las habilidades argumentativas a través de la preparación del vídeo para la campaña publicitaria (pregunta $n .^{\circ} 7$ ) ha sido puntuada con 5 estrellas por 5 encuestados, con 4 estrellas por tres de ellos, con 3 estrellas por 2 y uno de los participantes no ha contestado esta pregunta; a la pregunta de si los métodos empleados para la preparación de la fase oral han sido adecuados al grado de exigencia de las rondas orales (pregunta n. ${ }^{\circ} 8$ ) 7 estudiantes han otorgado una valoración de 5 estrellas, 2 de 4 estrellas y 2 de 3 estrellas; por su parte, la capacidad para comunicarse de manera clara y eficaz, que ha mejorado la confianza del alumnado para hablar en público (pregunta $\mathrm{n}^{\circ}$ 9) ha sido puntuada con 5 estrellas por 6 de los encuestados y con 4 estrellas por 5 de ellos; la mejora general de la capacidad para expresarse oralmente (pregunta n. $^{\circ}$ 10) ha obtenido 7 puntuaciones de 5 estrellas, 3 de 4 estrellas y 1 de 3 estrellas; finalmente, la percepción de los participantes sobre si las estrategias para la comunicación oral aprendidas les servirán en la vida académica y profesional (pregunta n. ${ }^{\circ}$ 11) ha sido valorada con 5 estrellas en 7 casos y con 4 estrellas en 4 casos. Los resultados obtenidos se reflejan de forma gráfica en la Figura 1, en la que la escala de 1 
a 11 identifica las preguntas formuladas en el cuestionario y la escala de 1 a 5 la puntuación otorgada.

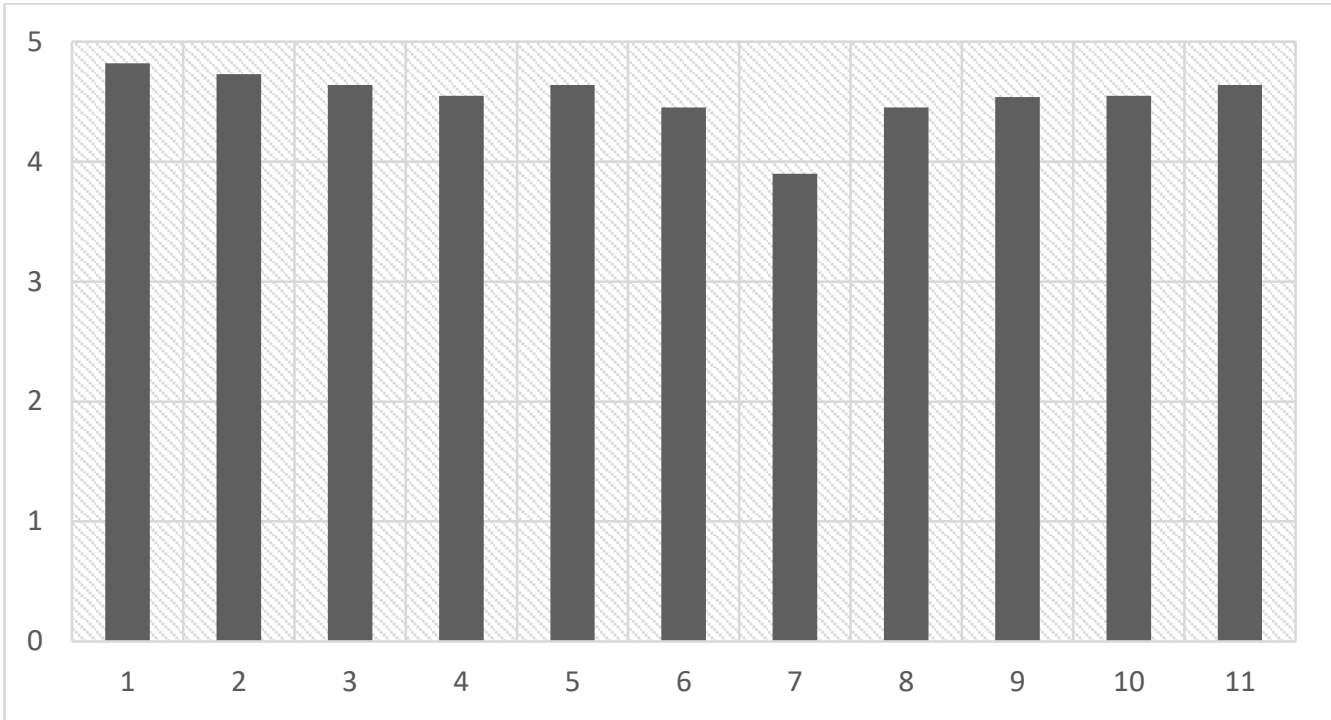

Figura 1. Resultados generales

Fuente: Elaboración propia

No obstante, como se ha señalado, estos resultados contrastan con aquellos de uno de los cuestionarios, en el que se atribuye una puntuación de 3 estrellas a la pregunta $n .^{\circ} 1$, de 2 estrellas a las preguntas n. ${ }^{\text {os }} 2,3,4$ y 6 , y de 1 estrella a las preguntas $n .^{\text {os }} 5,8,9,10$ y 11. La pregunta n. 7 no se contesta (Figura 2, idéntica leyenda que la Figura 1). Parece oportuno, por tanto, indagar e intentar comprender a qué se debe tal discrepancia.

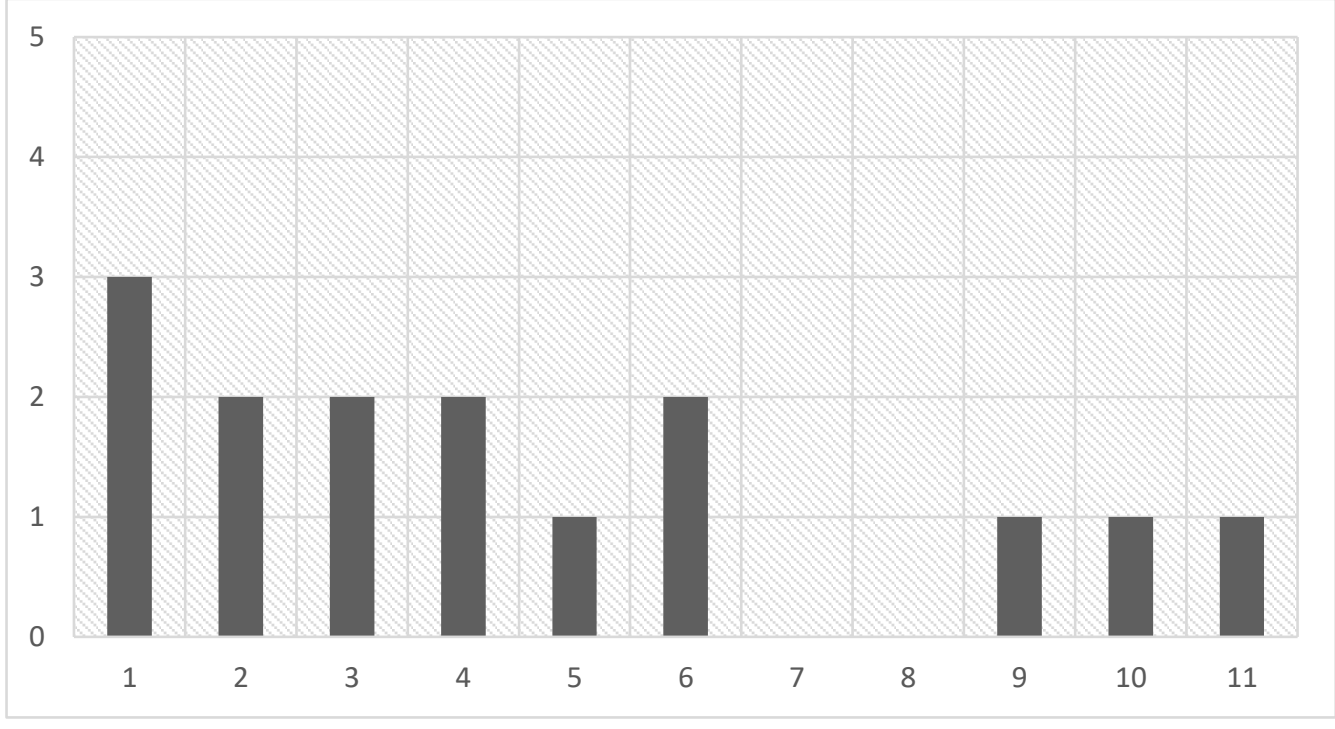


Figura 2. Resultados discrepantes

Fuente: Elaboración propia 


\subsection{Valoración de los resultados}

La valoración de los resultados obtenidos debe partir de la premisa de que la muestra obtenida, de tan solo 12 cuestionarios contestados, es bastante reducida. Sin duda, hubiese sido deseable contar con un número más amplio de opiniones. No obstante, sigue siendo un instrumento útil a la hora de elaborar estrategias docentes de cara a ediciones próximas.

Pues bien, en términos generales, se constata el alto grado de satisfacción de los alumnos participantes en la competición de arbitraje MOOTmadrid en relación con la adquisición y mejora de una serie de competencias relacionadas con el desarrollo de la oratoria jurídica, tanto a nivel de identificación, análisis y solución de problemas jurídicos, como de organización del discurso oral, interacción oral y argumentación, de gestión del tiempo en las intervenciones orales, trabajo bajo presión y comunicación clara y eficaz ante un auditorio. Todo ello ha redundado en la mejora sensible de la confianza del alumnado para hablar en público y de su capacidad para expresarse oralmente, aptitudes que resultan necesarias en su vida académica y profesional. En efecto, así lo ponen de manifiesto los resultados generales reflejados en la Figura 1, donde puede observarse una media superior a 4 puntos en la mayoría de las preguntas contestadas. En este sentido, también es revelador el comentario incluido por uno de los alumnos en la encuesta, pues afirma que «[l] a participación en el MOOTmadrid ha sido una experiencia muy enriquecedora para mí. La preparación que recibimos por parte de los profesores nos ha permitido trabajar y mejorar notablemente nuestras habilidades de expresión, redacción y argumentación jurídicas, tan necesarias para el ejercicio del Derecho. Además, pudimos ver la complejidad que puede alcanzar un caso y la importancia que tiene aportar soluciones rigurosas a cada problema que se plantea sobre el mismo. También, al menos a mí, la formación recibida estos meses me ha hecho ganar confianza a la hora de hablar y debatir en público sobre cuestiones tan complicadas como las que se trataban en las audiencias, y mejorar mi oratoria y capacidad de reacción ante las preguntas de un tribunal».

No obstante, sería contraproducente para los futuros participantes en la competición si perdiéramos de vista que la puntuación otorgada en una de las preguntas, la n. ${ }^{\circ} 7$, referente a la preparación de la campaña publicitaria, ha sido ligeramente inferior a 4 
puntos. Sin duda, ello hace necesario dedicar especial esfuerzo a mejorar este aspecto de cara a ediciones futuras, según se propone en el siguiente punto del presente trabajo.

Por lo que respecta ahora a los resultados discrepantes con el sentir general, expresados por tan solo uno de los encuestados (Figura 2), hay que decir que los mismos quedan relativizados por su excepcionalidad, esto es, su ausencia de concordancia con las respuestas obtenidas en todos los restantes cuestionarios, que sí merecen ser consideradas fiables, pues revisten las características de homogeneidad y coherencia, tanto interna - es decir, dentro de un mismo cuestionario las calificaciones otorgadas no son discordantes - como de conjunto, por cuanto todos los demás participantes valoran de manera similar los ítems encuestados. Así se deduce también del propio comentario insertado por el alumno en el espacio destinado al efecto, en el que afirma que:

«[b]ajo mi punto de vista, se trata de un evento con alto grado competitivo, que me gustó experimentar pero que a nivel personal no me aportó tanto como esperaba. Dado que si no eres el que participa en las rondas orales no tienes mucho que hacer. Hubiera estado mejor que hubiésemos tenido varios equipos de demandantes y demandados para poder participar más, compartir la experiencia entre compañeros y aprender unos de otros, ya que en ocasiones hay fallos o aspectos que no ves en ti mismo y si en los demás. Está bien ser competitivo, pero me parece que lo que debería primar aquí, puesto que al participar en el MOOT suele ser la primera toma de contacto con el mundo que más se asemeja a lo laboral, debería ser fundamental aprender y formarse, más que ganar».

En efecto, basta con remitirse al punto 3 precedente, referente al desarrollo y la preparación de la fase oral del MOOTmadrid, para comprender que la participación en la competición exige que todos los alumnos se involucren activamente en la preparación de la fase oral, no sólo aquellos elegidos democráticamente para debatir en dicha fase. En consecuencia, es probable que la afirmación de que «si no eres el que participa en las rondas orales no tienes mucho que hacer» se deba a la falta de motivación del alumno en cuestión, que le ha llevado a no intervenir en el proceso de aprendizaje o al menos a intervenir de forma pasiva. Al respecto, debe destacarse que la participación en la competición es voluntaria para los alumnos y que, además, éstos deben superar un riguroso proceso de selección, pues el número de candidatos suele ser superior a aquel necesario para formar el equipo. A pesar de ello, puede ocurrir que el nivel de trabajo exigido a los participantes exceda de sus expectativas, lo que en algún caso puntual deriva en pérdida de la motivación. Es por ello por lo que en adelante se abordarán 
estrategias para incrementar el componente motivacional, especialmente en la preparación de la fase oral, a fin de que todos los participantes tomen conciencia de que el resultado obtenido es del equipo, y no solo de los ponentes en la fase oral, y que, en consecuencia, las actividades de preparación requieren de la implicación de todos los participantes.

\section{Conclusiones y propuestas de mejora}

A la vista de los resultados obtenidos en las encuestas no hay duda de que la participación en la competición de arbitraje MOOTmadrid constituye una herramienta didáctica adecuada para fomentar el desarrollo de la oratoria jurídica de los estudiantes del Grado en Derecho de la Universitat Jaume I, pues les ha permitido adquirir y mejorar competencias necesarias para expresarse ante un auditorio, de acuerdo con las exigencias propias de la disciplina jurídica. Como se ha señalado, los resultados obtenidos en la pregunta n. 7 han sido levemente inferiores a los de las demás preguntas. Por ello, sería conveniente recurrir a la colaboración de algunos alumnos de otras titulaciones como, por ejemplo, el Grado en Comunicación Audiovisual de la Universitat Jaume I, preferiblemente de tercer o cuarto curso, para que asesoren tanto a los docentes como al alumnado en la preparación de la campaña publicitaria.

Asimismo, debe destacarse que, a pesar de los excelentes resultados generales obtenidos en la encuesta, los resultados reales obtenidos en la fase oral del MOOTmadrid son y han sido modestos a lo largo de las distintas ediciones en las que nuestra universidad ha participado en la competición. Por tanto, las sucesivas mejoras que se han ido introduciendo con los años para optimar tanto la transmisión de conocimientos entre los miembros del equipo, de modo que cada participante pudiera adquirir una comprensión global del caso y no solo del tema que ha preparado, como las habilidades de oratoria de los estudiantes, mediante el aumento de ensayos preparatorios de la fase oral, no han dado los frutos esperados hasta ahora.

Esta circunstancia hace necesario plantear nuevas estrategias didácticas encaminadas a mejorar las competencias orales de los estudiantes. Para ello, se proponen otras dos mejoras. En primer término, y siguiendo las sugerencias realizadas en la encuesta, se plantea asignar como nueva tarea en el equipo la visualización de las rondas orales finales de las últimas ediciones de la competición, cuya grabación suele estar disponible en su página web. Su visionado y posterior comentario permitirá a los alumnos 
familiarizarse con la puesta en escena y desarrollo de las audiencias, además de proporcionarles nuevas herramientas para mejorar sus habilidades de comunicación oral. En segundo término, se propone acercar en la medida de lo posible el ensayo de la fase oral al desarrollo real de las rondas orales de la competición. A tal fin, parece oportuno plantear que algunos de los ensayos se realicen delante de un auditorio formado por distintos profesores, profesionales y alumnos que sean ajenos a los miembros del equipo y, además, intervengan durante los debates de forma espontánea, formulando distintas cuestiones para así emular a los árbitros durante la competición. En este marco, también parece adecuado que el debate, que rige la revisión de las actuaciones de los estudiantes durante el ensayo de las rondas orales, se estructure intentando replicar con la mayor fidelidad posible el tipo de discusiones que se llevan a cabo durante la competición. 
Anexo único

\section{ENCUESTA UJI SOBRE LA PARTICIPACIÓN EN EL MOOTMADRID}

Por favor, contesta las siguientes preguntas en una escala de 1 a 5 , teniendo en cuenta que "1" significa "nada" y "5" significa "totalmente".

1. La participación en el MOOTmadrid ha mejorado mi capacidad para identificar, analizar y resolver situaciones prácticas desde un punto de vista jurídico.

\begin{tabular}{|l|l|l|l|l|}
\hline 1 & 2 & 3 & 4 & 5 \\
\hline & & & & \\
& & & & \\
\hline
\end{tabular}

2. La participación en el MOOTmadrid ha mejorado mi capacidad para organizar el discurso oral.

\begin{tabular}{|l|l|l|l|l|}
\hline 1 & 2 & 3 & 4 & 5 \\
\hline & & & & \\
& & & & \\
\hline
\end{tabular}

3. La participación en el MOOTmadrid ha mejorado mi capacidad de interacción oral.

\begin{tabular}{|l|l|l|l|l|}
\hline 1 & 2 & 3 & 4 & 5 \\
\hline & & & & \\
\hline
\end{tabular}

4. La participación en el MOOTmadrid ha mejorado mi capacidad de argumentación.

\begin{tabular}{|l|l|l|l|l|}
\hline 1 & 2 & 3 & 4 & 5 \\
\hline & & & & \\
& & & & \\
\hline
\end{tabular}

5. La participación en el MOOTmadrid ha mejorado mi capacidad para gestionar adecuadamente el tiempo en las intervenciones orales.

\begin{tabular}{|l|l|l|l|l|}
\hline 1 & 2 & 3 & 4 & 5 \\
\hline & & & & \\
\end{tabular}

6. La participación en el MOOTmadrid ha mejorado mi capacidad para trabajar bajo presión. 


\begin{tabular}{|l|l|l|l|l|}
\hline 1 & 2 & 3 & 4 & 5 \\
\hline & & & & \\
\hline
\end{tabular}

7. La preparación del vídeo para la campaña publicitaria ha mejorado mis habilidades argumentativas.

\begin{tabular}{|l|l|l|l|l|}
\hline 1 & 2 & 3 & 4 & 5 \\
\hline & & & & \\
\hline
\end{tabular}

8. Los métodos empleados para la preparación de la fase oral han sido adecuados al grado de exigencia de las rondas orales.

\begin{tabular}{|l|l|l|l|l|}
\hline 1 & 2 & 3 & 4 & 5 \\
\hline & & & & \\
& & & & \\
\hline
\end{tabular}

9. La participación en el MOOTmadrid me ha permitido adquirir una comunicación clara y eficaz que ha mejorado mi confianza para hablar en público.

\begin{tabular}{|l|l|l|l|l|}
\hline 1 & 2 & 3 & 4 & 5 \\
\hline & & & & \\
\hline
\end{tabular}

10. En general, la participación en el MOOTmadrid ha mejorado mi capacidad para expresarme oralmente.

\begin{tabular}{|l|l|l|l|l|}
\hline 1 & 2 & 3 & 4 & 5 \\
\hline & & & & \\
& & & & \\
\hline
\end{tabular}

11. Las estrategias para la comunicación oral aprendidas me servirán en la vida académica y profesional.

\begin{tabular}{|l|l|l|l|l|}
\hline 1 & 2 & 3 & 4 & 5 \\
\hline & & & & \\
\hline
\end{tabular}


A continuación, puedes escribir brevemente cualquier otro comentario sobre tu participación en el MOOTmadrid:

\section{Referencias bibliográficas}

Agencia Nacional de Evaluación de la Calidad y Acreditación (ANECA) (2005): Libro Blanco del Título de Grado en Derecho, http://www.aneca.es/var/media/150240/libroblanco_derecho_def.pdf (fecha última consulta: 24 de noviembre de 2019).

ARMENGOT VILAPLANA, A. (2016): «La metodología docente de la simulación de un proceso». En M. Turull Rubinat y E. Albertí Rovira (Eds.) 74 experiencias docentes del Grado en Derecho. Barcelona: Octaedro, Universitat de Barcelona, Institut de Ciències de l'Educació, ICE (pp. 33-36).

ARROYO VENDRELL, T. (2016): «La implementación de las experiencias moots en el grado en derecho». En M. Turull Rubinat y E. Albertí Rovira (Eds.) 74 experiencias docentes del Grado en Derecho. Barcelona: Octaedro, Universitat de Barcelona, Institut de Ciències de l'Educació, ICE (pp. 37-40).

Bases y reglas de la Competición Internacional de Arbitraje y Derecho mercantil, Universidad Carlos III de Madrid, España, http://www.mootmadrid.es/2019/files/2012/05/REGLAS-MOOT-MADRID-4.pdf (fecha última consulta: 24 de noviembre de 2019).

BOBOC, S. (2018): «La participación de los estudiantes del grado en Derecho en la competición internacional de arbitraje y derecho mercantil como herramienta para la adquisición de competencias propias de la titulación. Docencia y Derecho». Revista para la docencia jurídica universitaria, número 12, http://www.uco.es/docencia_derecho/index.php/reduca/article/view/148 (fecha última consulta: 24 de noviembre de 2019).

CAÑABETE PÉREZ, J. (2016): «La realización de una liga de debate y argumentación como método de aprendizaje en una asignatura instrumental». En M. Turull Rubinat y 
E. Albertí Rovira (Eds.) 74 experiencias docentes del Grado en Derecho. Barcelona: Octaedro, Universitat de Barcelona, Institut de Ciències de l’Educació, ICE (pp. 53-56).

DE MIGUEL, M. (2006): «Métodos y modalidades de enseñanza en la educación superior». En M. de Migel (Coord.) Metodologías de enseñanza y aprendizaje para el desarrollo de competencias. Orientaciones para el profesorado Universitario ante el Espacio Europeo de Educación Superior, Madrid: Alianza Editorial (pp. 17-26).

DÍAZ PITA, M. ${ }^{a}$ P. (2016): «Los juicios simulados como técnica de aprendizaje colaborativo y de evaluación continua». En M. Turull Rubinat y E. Albertí Rovira (Eds.) 74 experiencias docentes del Grado en Derecho. Barcelona: Octaedro, Universitat de Barcelona, Institut de Ciències de l'Educació, ICE (pp. 97-100).

GABRIEL, H. D. (1999): Preparation and Delivery of Oral Argument in Appellate Courts. American Journal of Trial Advocacy, vol. 22 (pp. 571-590).

GARCÍA AÑÓN, J. (2014): «La integración de la educación jurídica clínica en el proceso formativo de los juristas». Revista de Docencia Universitaria, vol. 12, número extraordinario 3 (pp. 153-175).

GOÑI, M. Y MESEGUER, S. (2010): «Diseño curricular centrado en las competencias que debe adquirir el estudiante del Grado en Derecho». Formación Universitaria, vol. 3, número 10 (pp. 37-46).

GRAVES, J. M. Y VAUGHAN, S. A. (2006): «The Willem C. Vis International Commercial Arbitration Moot: Making the most of an Extraordinary Educational Opportunity». Vindobona Journal of International Commercial Law and Arbitration, número 10 (pp. 173-206).

MARTINEAU, R. J. (1989): «Appellate Litigation: Its Place in the Law School Curriculum». Journal of Legal Education, vol. 39, número 1 (pp. 71-86).

Memoria del Título de Graduado o Graduada en Derecho por la Universitat Jaume I (2013), https://documents.uji.es/alfresco/d/d/workspace/SpacesStore/bbc80171-53e846a8-be50-

782ca1c83c1e/Memoria+modificada+por+ANECA+261213+Derecho.pdf?guest=true (fecha última consulta: 24 de noviembre de 2019). 
PERALES VISCASILLAS, P. (2016): «Participación en concursos internacionales (Moot Viena)». En M. Turull Rubinat y E. Albertí Rovira (Eds.) 74 experiencias docentes del Grado en Derecho. Barcelona: Octaedro, Universitat de Barcelona, Institut de Ciències de l'Educació, ICE (pp. 251-254).

PUETZ, A. (2015): «La participación en competiciones de arbitraje como herramienta didáctica en la enseñanza del Derecho». En V Jornada Nacional sobre estudios universitarios y I Taller de Innovación Educativa. Univeristat Jaume I. Castellón: $\begin{array}{llll}\text { Universitat Jaume } & \text { I } & \text { 396-406), }\end{array}$ http://www.tenda.uji.es/pls/iglu/!GCPPA00.GCPPR0002?lg=CA\&id_art=1470 (fecha última consulta: 24 de noviembre de 2019).

RACHID, M. A. Y KNERR, CH. R. (2000): «Brief History of Moot Court: Britain and U.S». Presentación en la Annual Meeting of the Southwestern Political Science Association. Galveston, http://eric.ed.gov/?id=ED442343 (fecha última consulta: 24 de $\underline{\text { noviembre de 2019). }}$.

RAMÍREZ RAMÍREZ, J. (2002): «La expresión oral». Contextos educativos, número 5 (pp. 57-72).

SÁNCHEZ SÁNCHEZ, A. (2016): «Enseñando desde la práctica y simulación de juicios». En M. Turull Rubinat y E. Albertí Rovira (Eds.) 74 experiencias docentes del Grado en Derecho. Barcelona: Octaedro, Universitat de Barcelona, Institut de Ciències de l'Educació, ICE (pp. 293-296).

VEGA VEGA, J. A. (2009): «Metodología docente en derecho mercantil». Revista de estudios económicos y empresariales, número 20 (pp. 29-69).

VILLA, A. Y POBLETE, M. (2007): Aprendizaje basado en competencias. Una propuesta para la evaluación de las competencias genéricas. Bilbao: Ediciones Mensajero. 